\title{
Comparative Analysis of Performance of Phase Coded Pulse Compression
} \section{Techniques}

\author{
P. Keerthi Silpa ${ }^{1}$, N. Deepika Rani ${ }^{2}$ and N. Bala Subrahmanyam ${ }^{3}$
}

\begin{abstract}
RADAR performance is measured by its sensitivity of detecting targets at farther distance and range resolution capability. To improve the detection ability long pulse is needed and to improve range resolution capability short pulse is needed. Thus, there exists trade-off between detection and range resolution. To overcome this problem, pulse compression techniques are employed. Pulse compression can be done either by using frequency modulation or phase modulation to simple pulse. Utilization of frequency modulated pulse compression techniques leads to arousal of high side-lobes at the matched filter output. Presence of high sidelobes leads to target masking in presence of multiple targets. To reduce peak side-lobe levels phase coded pulse compression technique is employed. In this paper, the PSL of various phase coding techniques are compared through simulations in LabVIEW.
\end{abstract}

Keywords - Matched Filter, Range Resolution, Phase Codes, PSL

\section{INTRODUCTION}

RADAR is used in military for surveillance, controlling and guiding weapons, for navigation of ship etc. In many of these applications, system employs pulse compressed waveform good performance. Lewis B.L. and Kretschmer F.F. [1]-[2] describes the evolution of Frank, P1, P2, P3 and P4 phase codes from LFM and summarized on the derivation of poly-phase codes. Rawat C.D. and Sarate A.D. [3] compared peak side-lobe level, range resolution of both frequency and phase modulated pulse compression techniques simulating in MATLAB. The importance of matched filter in the receiver section for detecting the presence of the targets demonstrated by Skolnik M.I. [4]. Eustice D., Baylis C. and Marks R. J. [6] described and detailed the properties of Woodward's ambiguity function which is output of matched filter.

In this paper, implementation of matched filter is described in section II. Dependency of range resolution on pulse width is illustrated in section III. Various phase coding techniques and corresponding matched filter responses are explained in section IV. The work is summarized in in section V by comparing the PSL of various phase codes.

\section{MATCHED FiLTER}

Basic operations performed by RADAR in the receiver section is detecting the presence of the target. Matched filter is the basic important block in the receiver section whose output is used to extract information and to detect the presence of target. Matched filter is a network whose frequency-response function leads to maximum output peak signal to mean noise power ratio (SNR). It is a linear time invariant (LTI) system and causal system whose impulse response determined maximizes the SNR. In the receiver section, the received echo signal is demodulated therefore signal

\footnotetext{
${ }^{1}$ Department of Electronics and Communication Engineering Gayatri Vidya Parishad College of Engineering (Autonomous), Visakhapatnam, A.P, India

${ }^{2}$ Department of Electronics and Communication Engineering Gayatri Vidya Parishad College of Engineering (Autonomous), Visakhapatnam, A.P, India

${ }^{3}$ Department of Electronics and Communication Engineering Gayatri Vidya Parishad College of Engineering (Autonomous), Visakhapatnam, A.P, India
} 
processing is done on the envelope of the echo. Therefore, the input to matched filter is only envelope of the echo signal. If $s(t)$ is the input to the matched filter, then the impulse response of matched filter $h(t)$ is given as [4],

\section{$h\left(t^{2}\right)=z^{9}\left(t_{\mathrm{m}}-t^{2}\right)$}

where $t_{m}=$ the time instant where maximum SNR is obtained. Then the output of the matched filter is obtained by convolving the input $\sigma(\underline{t})$ with impulse response of the matched filter $\hat{a}(\boldsymbol{t})$

\section{$y(t)=s\left(t^{2}\right)+\varepsilon^{2}\left(t_{n}-t\right)$}

For real signals $v^{\circ}(t)=g(t)$. Then the response of the matched filter is equivalent to cross correlation between envelope of received and transmitted signal. Implementation of the filter matched to simple pulse $s(t)$ of unit amplitude extending from 0 to $\tau=10 \mu \mathrm{s}$ is shown in the Figure 1 .

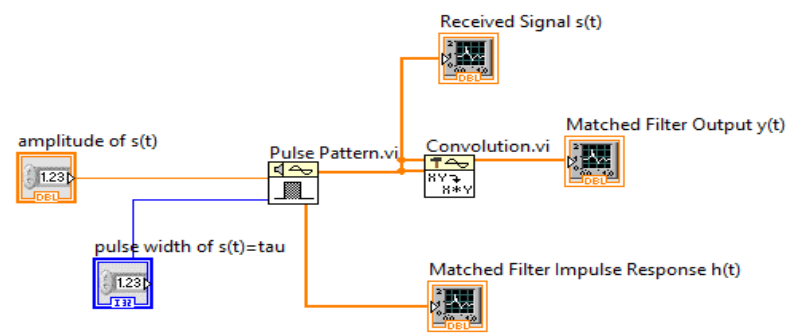

Figure 1. Block diagram of filter which matched to simple pulse of width

In Figure 1, Pulse pattern.vi is used to generate pulse of $10 \mu$ s width and unit amplitude. Matched filter response is obtained Convolution.vi. For a target at range $R_{0}$, corresponding round trip time delay $\boldsymbol{t}_{0}$, the complex envelope of echo received will be $s\left(t-t_{0}\right)$ instead of $s(t)$. Now $s\left(t-t_{D}\right)$ is fed as input to the filter matched to $s(t)$ as shown in Figure 2(a).
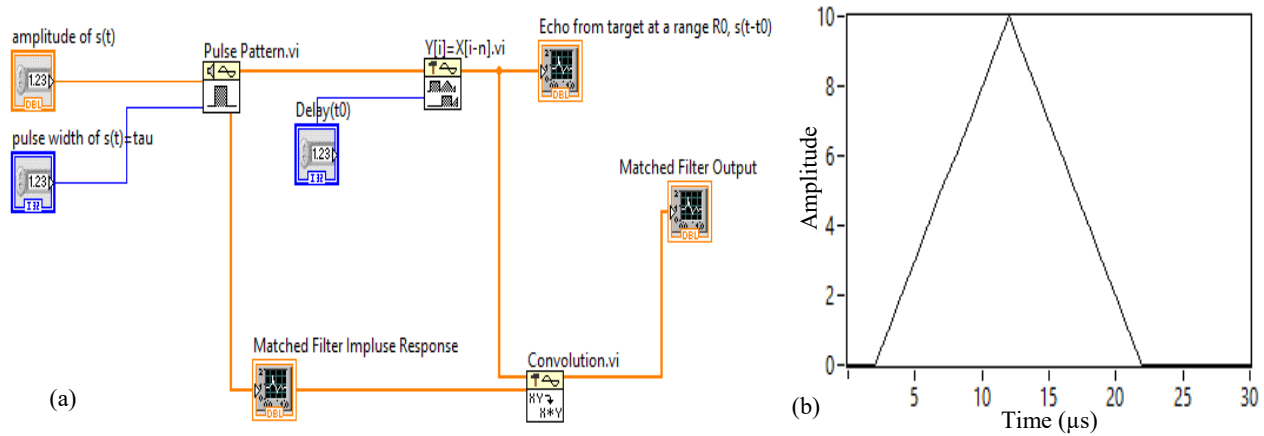

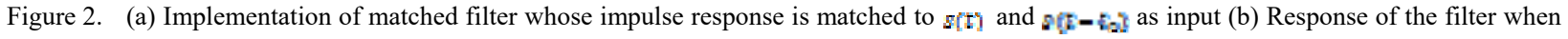
input is with delay tion 20

In Figure 2(a), $\mathrm{Y}[\mathrm{i}]=\mathrm{X}[\mathrm{i}-\mathrm{n}] . \mathrm{vi}$ is used to delay $\sigma(\mathrm{t})$ by $2 \mu \mathrm{s}$. From Figure 2(b), it is observed that peak occurs at $12 \mu \mathrm{s}$. The range $(R)$ of the target from RADAR is calculated by using

$R=\frac{\text { ctipeshery }}{2}$

is the time instant where peak occurs in the matched filter output. Then the range $\left(R_{D}\right)$ calculated using eq. (3) is obtained as $0.3 \mathrm{~km}$.

\section{Relation between Range Resolution and Pulse Width}

Range resolution [5] is one of the useful metric that describes the ability of RADAR to detect the targets as distinct objects even if they are spaced closely. The RADAR is able to resolve two targets if and only if separation $(A R)$ between two targets is equal to $\frac{\pi \tau}{2}$.

Range resolution $(S R)=\frac{G 7}{2}$ 
where $c=$ velocity of light $=3 \times 10^{8} \mathrm{~m} / s, \tau=$ pulse width of the transmitted signal. Therefore, Range resolution is proportional to pulse width.

In order to examine the range resolving capability of radar, consider two targets at ranges $\mathbb{R}_{0}$ and $\mathbb{R}_{1}$ which are separated by $\Delta R$. For the simple pulse of duration $\tau=10_{0 R}$, the resolving capability of radar in range dimension is $1.5 \mathrm{~km}$ according to eq.(4).Assuming one target is fixed at range $R_{0}=0.3 \mathrm{~km}$ and other target position $R_{1}$ is varied accordingly to verify the range resolution eq. (4). Based on the position of second target, three different cases are seen. For these cases, composite filter responses are shown in Figure 3, Figure 4 and Figure 5, where (a) indicates composite response obtained if individual target responses are added in phase and (b) indicates composite response obtained when target responses are added out of phase.

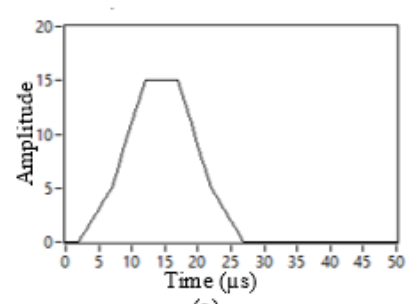

(a)

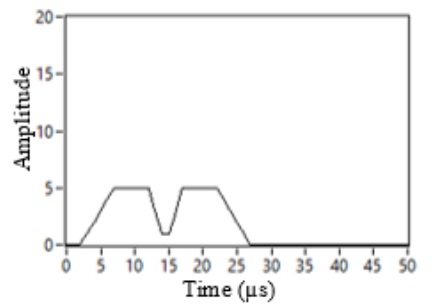

(b)

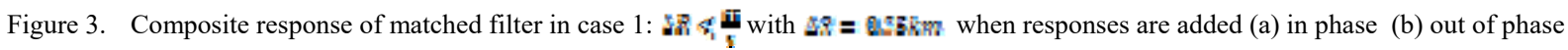

From Figure 3(a), a trapezoid with small flat region is seen but from Figure 3(b), user is unable to detect the presence of targets since peaks are degraded here. Similarly in case $2: \Delta R=\frac{\pi}{2}, \Delta R$ is chosen to be $1.5 \mathrm{~km}$. Then the composite responses are presented in the Figure 4.
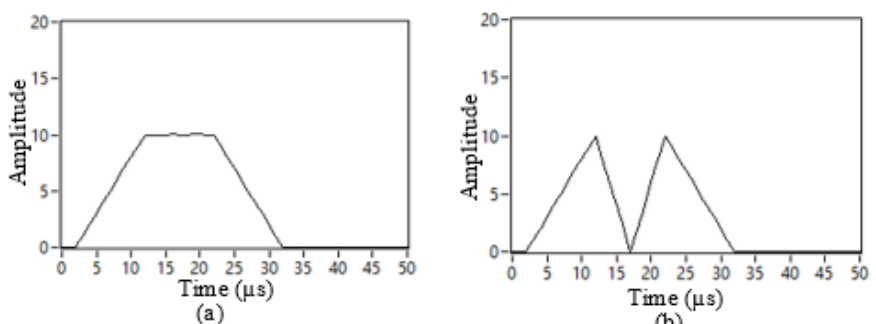

(b)

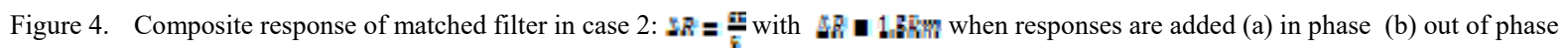

In Figure 4(a) trapezoid with flat region extended for more duration compared to case 1 is observed and in Figure 4(b) two separate peaks are observed which indicate the presence of two different targets. In the case $3: \Delta R \geq \frac{\pi}{\mathbb{a}}$, target 2 is at range $\mathbb{F}_{1}=3.3 \mathrm{Fm}$, the composite responses for this case are shown in Figure 5.
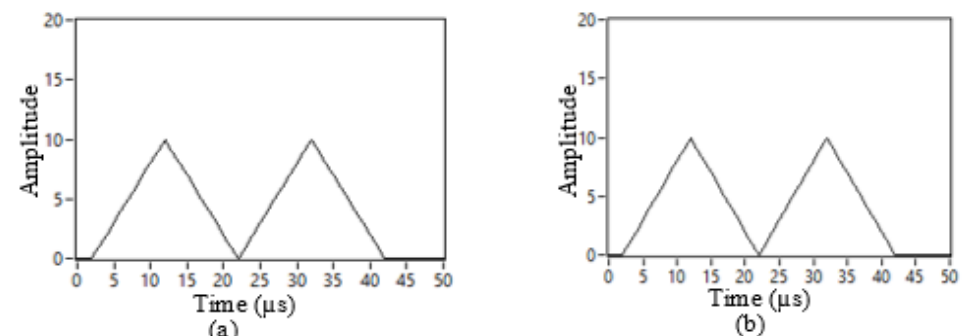

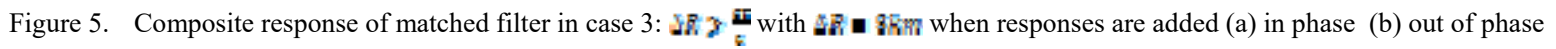

One can observe two peaks in Figure 5(a) and Figure 5(b) which indicates presence of two targets. In Figure 4(b) and Figure 5(b), two separate peaks are observed, it means targets are resolved as multiple. From these composite responses it is concluded that the minimum separation between targets must be at-least $\frac{\pi}{2}$ i.e. range resolution $(\Delta R)=\frac{\pi}{q}$. Pulse compression techniques are employed to resolve closely spaced targets. 


\section{Phase Coding Pulse Compression}

Phase modulation or phase coding [5] is an intra-pulse modulation technique in which simple pulse of width ( $v$ ) is divided into smaller pulses named as chips. Each chip is assigned a phase. Then, the envelope of phase coded waveform $s(t)$ can be modelled as collection of M continuous sub-pulses $s_{m}(t)$ each of duration $\tau_{c}$.

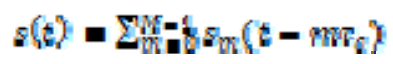

$s_{x}(t)= \begin{cases}6 / p_{n} & 0 \leq 5 \\ 0 & \text { elsewhere }\end{cases}$

where $\tau_{c}$ is referred to as chip duration, $\sigma_{\mathrm{m}}=$ phase of $\mathrm{m}^{\text {th }}$ chip. Hence, the total pulse width of phase coded waveform $(\tau)=M \tau_{\mathrm{r}}$. The number of chips is $\mathrm{M}$, renamed as length of the code.

\section{A. Bi-phase codes -}

In binary phase coding, $\Phi_{\mathrm{m}}$ can be either 0 or $\pi$. Therefore in case of binary phase coding, $\Sigma_{m}(t)$ takes values either +1 or -1 . Barker code belongs to family of binary phase codes. For 13-bit Barker code, the phase elements are given by $B_{18}=\{1,1,1,1,1,1,-1,1,1,1,1,1,1\}$. The response of filter matched to 13 bit barker code is obtained using eq. (2) with $t_{\mathrm{m}}=0$. Figure 6(a) represents the implementation of matched filter for Barker code with $t_{\mathrm{m}}=0$. AutoCorrelation.vi is used to perform auto-correlation for given input.
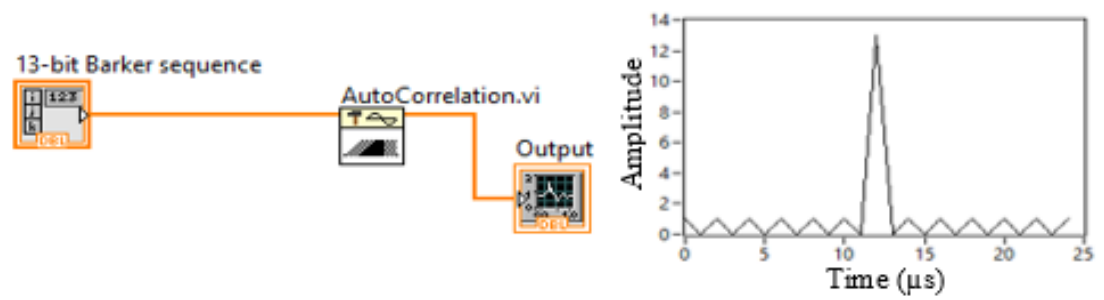

(a)

(b)

Figure 6. (a) Implementation of matched filter for 13-bit Barker code (b) Matched filter response for 13-bit Barker code

\section{B. Poly-phase codes -}

In The phase $\left\{\varphi_{n}\right\}$ can take any value within the interval $[0, \pi]$. Poly-phase codes that are used commonly are Frank Codes, P1, P2, P3, P4 codes.

\section{Frank code-}

The phase sequence is given by [1]

$$
\sigma_{\mathrm{min}}=\left(\frac{n_{\pi}}{N}\right)(m-1)(n-1)
$$

where $\mathrm{m}=1,2, \ldots \mathrm{N}$ and $\mathrm{n}=1,2, \ldots \mathrm{N}, \mathrm{N}$ is a positive integer, $M$ is the code sequence length chosen as $N^{2}$. Frank code of M-bit is generated using the eq. (5), eq. (6) and eq. (7). Matched filter implementation is shown in Figure 7(a). In Figure 7(a), $1 S_{\mathrm{m}}$ is given as input to 'Exponential block' to generate used to normalize the amplitude of the response.

D. P1 code-

The phase sequence is given by [1]

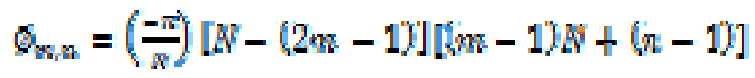

where $\mathrm{m}=1,2, \ldots \mathrm{N}$ and $\mathrm{n}=1,2, \ldots \mathrm{N}, \mathrm{N}$ is a positive integer, $M$ is the code sequence length chosen as $N^{2}$. P1 code of M-bit is generated using the eq. (5), eq. (6) and eq. (8) and matched filter implementation is shown in Figure. 8(a).

\section{E. $P 2$ code -}

The phase sequence is given by [1]

$$
S_{\mathrm{min}}=\left[\frac{\pi}{2}\left(\frac{N-4}{N}\right)-\frac{\pi}{N}(n-1)\right](P+1-2 m)
$$

where $\mathrm{m}=1,2, \ldots \mathrm{N}$ and $\mathrm{n}=1,2, \ldots \mathrm{N}, \mathrm{N}$ is a positive integer, $M$ is the code sequence length chosen as $N^{2}$. $\mathrm{P} 1$ code of M-bit is generated using the eq. (5), eq. (6) and eq. (9) and matched filter implementation is shown in Figure. 9(a). 


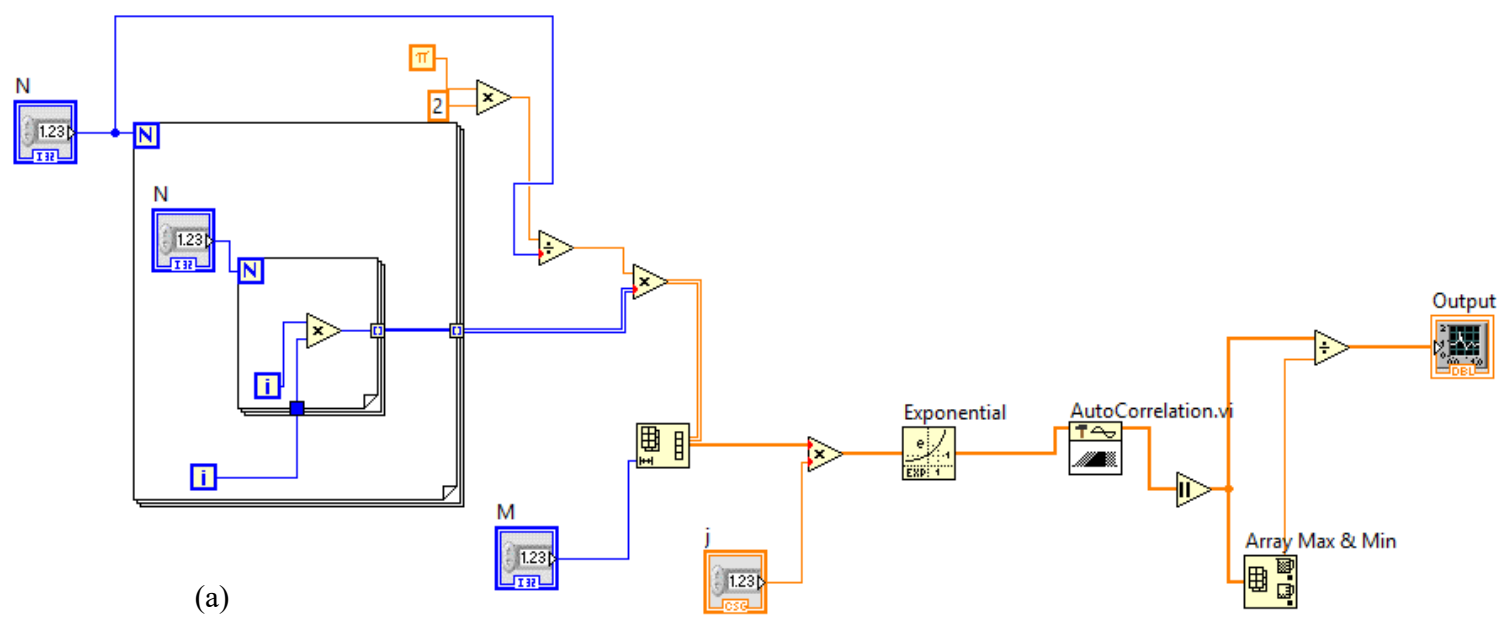

(b)

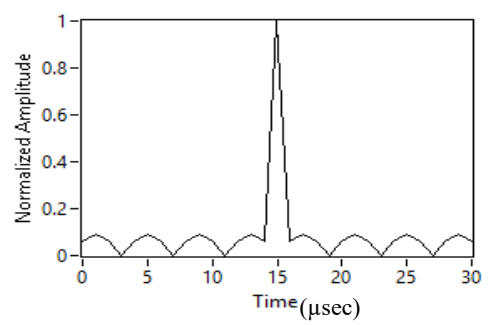

Figure 7. (a) Implementation of matched filter for M-bit Frank code (b) Matched filter response for 16-bit Frank code
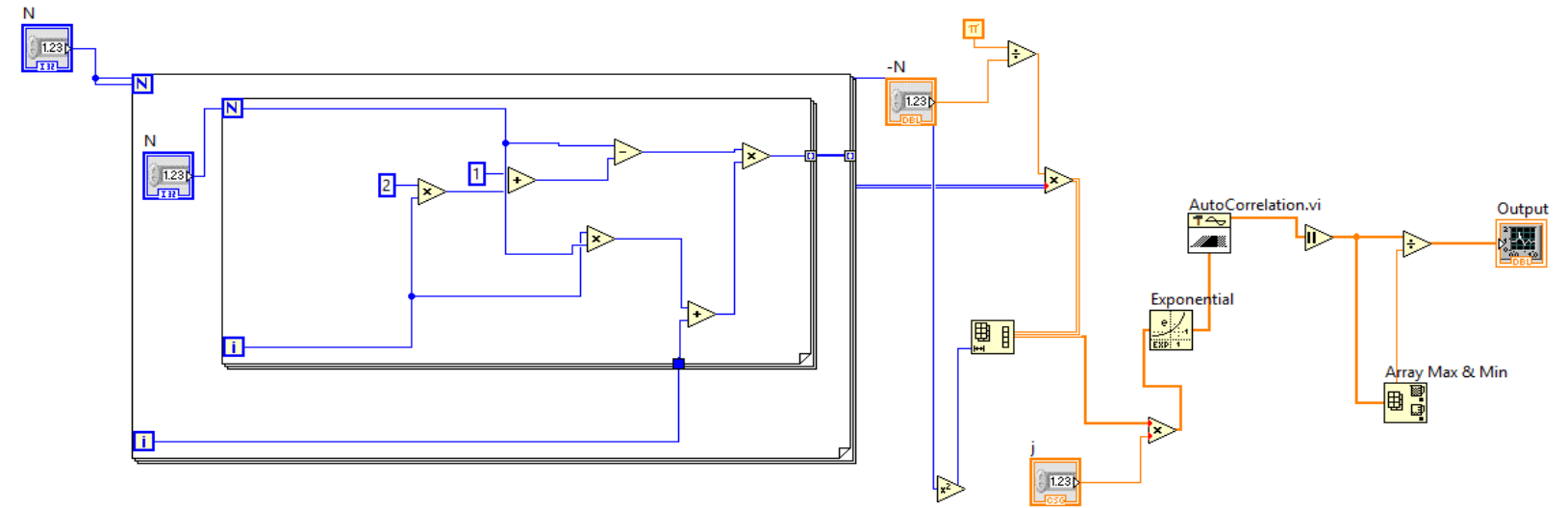

(a)

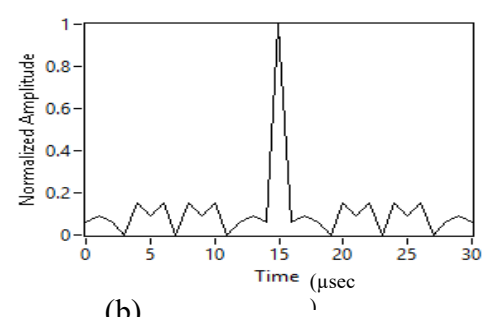

(b)

Figure 8. (a) Implementation of matched filter for M-bit P1 code (b) Matched filter response for 16-bit P1 code 


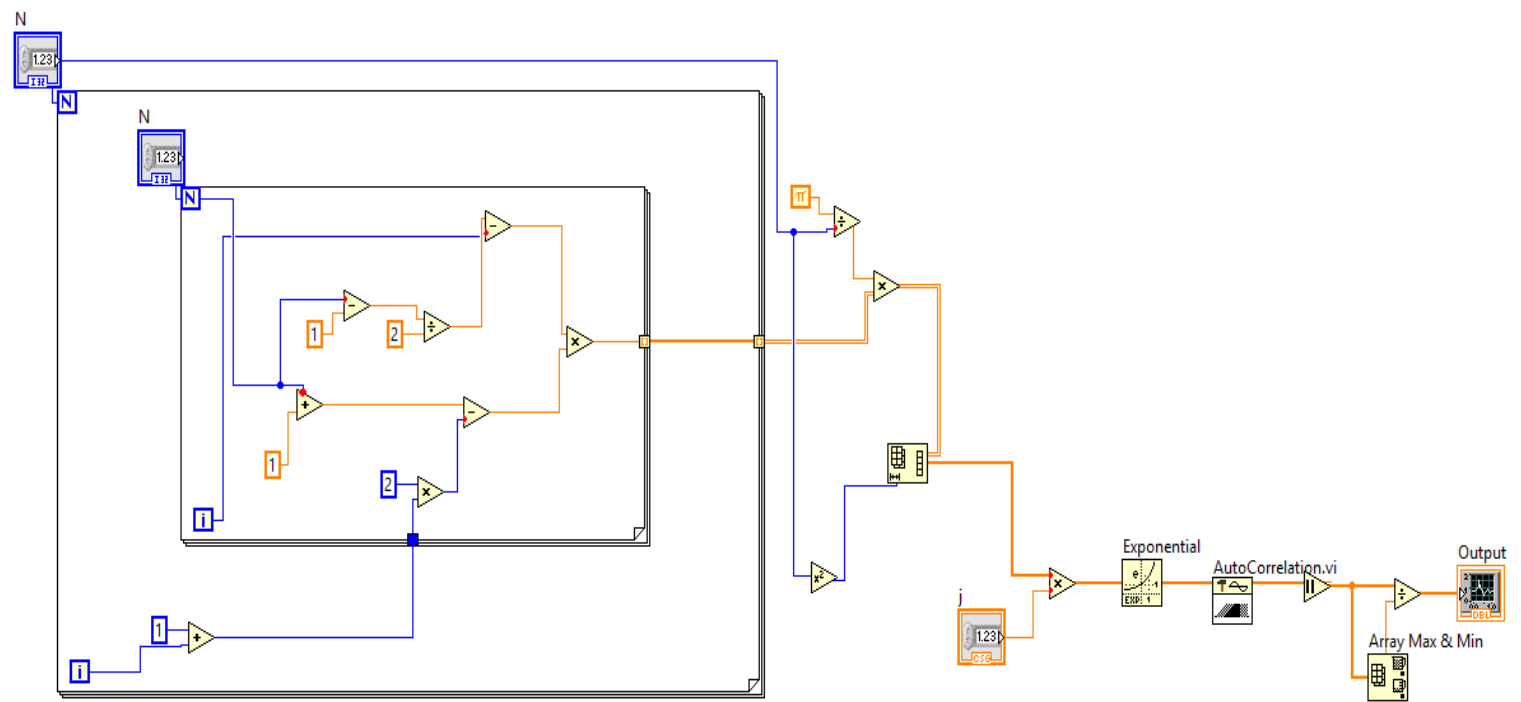

(a)

(b)

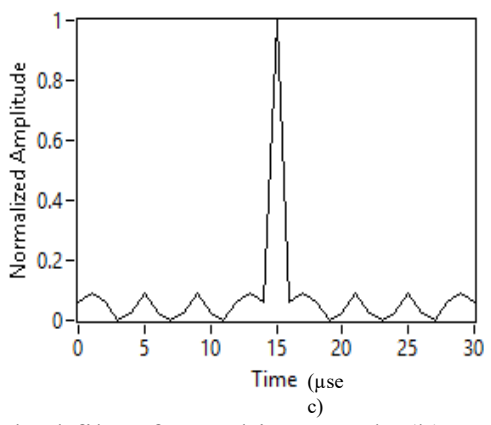

Figure 9. (a) Implementation of matched filter for M-bit P2 code (b) Matched filter response for 16-bit P2 code

F. P3 code-

The phase sequence is given by [2]

$$
\phi_{\mathrm{n}}=\left(\frac{\pi}{N}\right)(\mathrm{m}-1)^{2}
$$

where $\mathrm{m}=1,2, \ldots \mathrm{M}, \mathrm{M}$ is a positive integer that defines the code sequence length.P3 code of M-bit is generated using the eq. (5), eq. (6) and eq. (10) and implementation of matched filter is shown in fig. 10(a).

G. $P 4$ code -

The phase sequence is given by [2]

$$
\sigma_{n}=\left(\left(\frac{\pi}{w}\right)(m-1) 4\right)-\pi(m-1)
$$

where $\mathrm{m}=1,2, \ldots \mathrm{M}, \mathrm{M}$ is a positive integer that defines the code sequence length.P4 code of M-bit is generated using the eq. (5), eq. (6) and eq. (11) and implementation of matched filter is shown in Figure 11(a). 


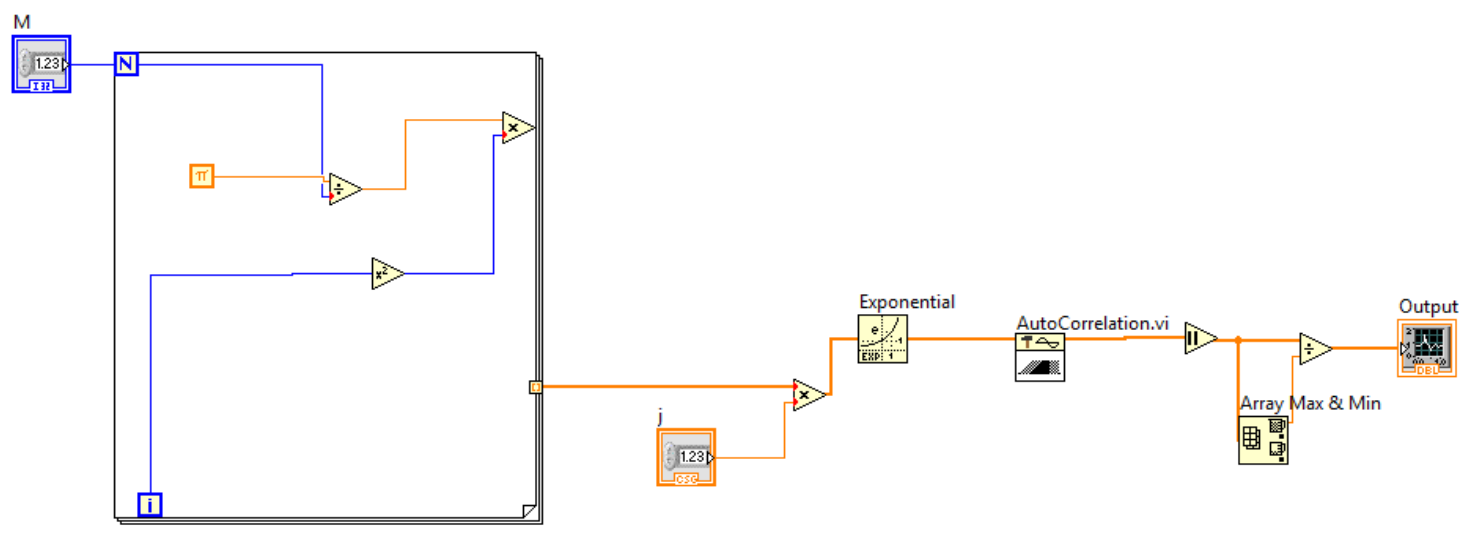

(a)

(b)

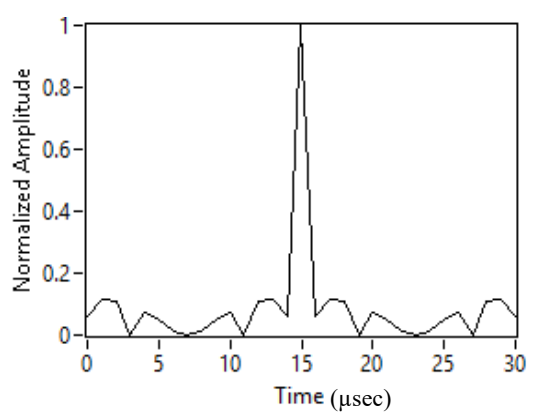

Figure 10. (a) Implementation of matched filter for M-bit P3 code (b) Matched filter response for 16-bit P3 code

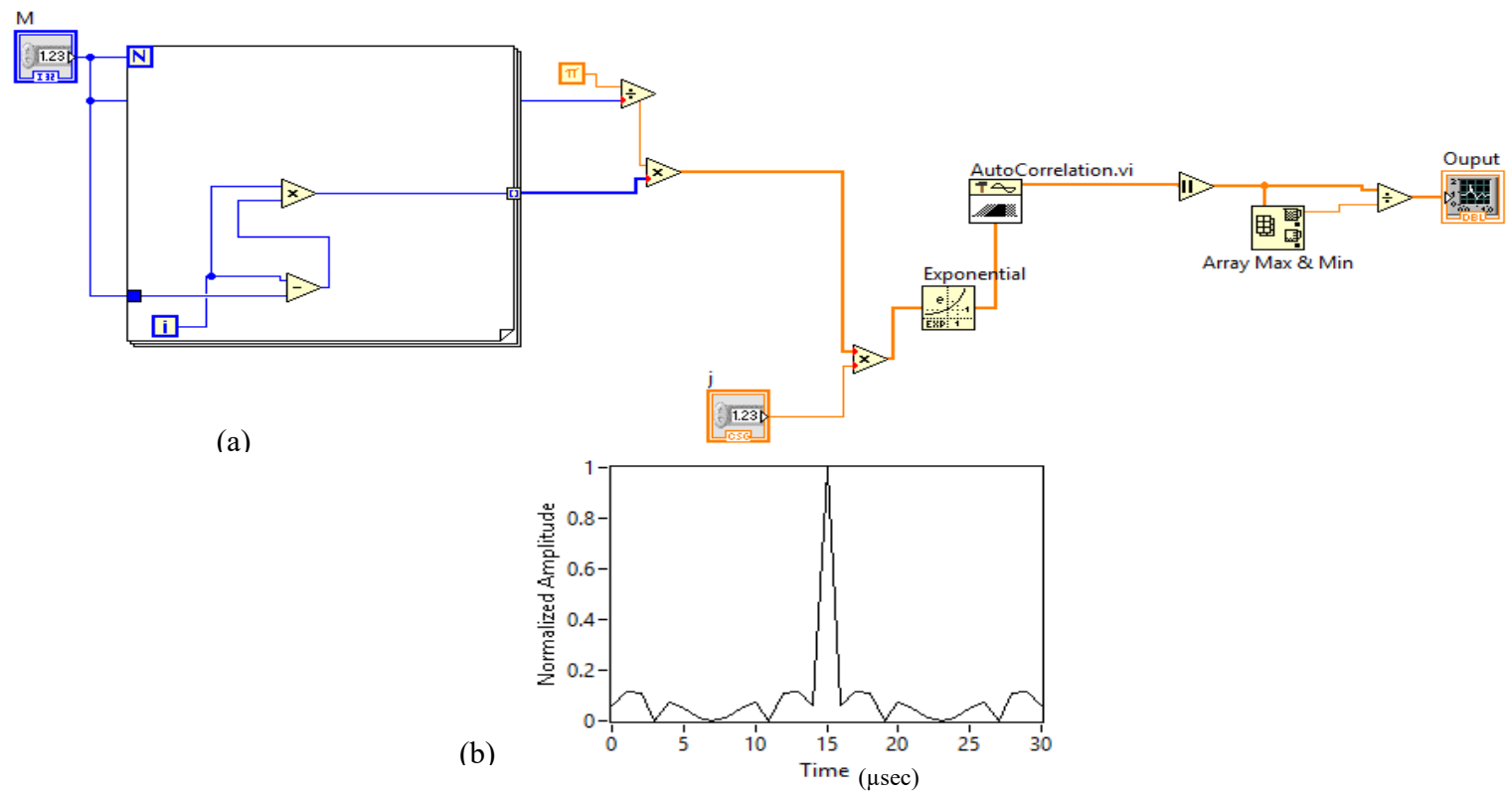

Figure 11. (a) Implementation of matched filter for M-bit P4 code (b) Matched filter response for 16-bit P4 code 


\section{IV.RESULTS AND DISCUSSIONS}

To compare the performance of the phase codes mentioned in the paper Peak Side-lobe Level (PSL) is chosen as metric. PSL is defined as ratio of peak side lobe amplitude to main lobe amplitude. PSL in dB is given by [3]

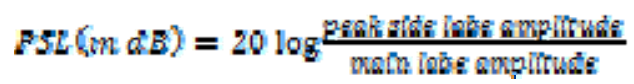

PSL of 13-bit Barker code $=-22.3 \mathrm{~dB}$. The major disadvantage of Barker codes is that they are limited in number [5]. Poly-phase codes like Frank code, P1 code, P2 code, P3 code and P4 codes are implemented for different lengths and corresponding PSL value of matched filter responses are tabulated in Table -1.

Table-1 Comparison of PSL for code lengths $\mathrm{M}=16, \mathrm{M}=64$ and $\mathrm{M}=100$

\begin{tabular}{|l|l|l|l|}
\hline Code & PSL(in dB) for M=16 & PSL(in dB) for M=64 & PSL(in dB) for M=100 \\
\hline Frank & -20.91 & -27.95 & -30.45 \\
\hline P1 & -16.47 & -21.93 & -24.43 \\
\hline P2 & -20.91 & -27.95 & -30.45 \\
\hline P3 & -19.17 & -24.43 & -26.02 \\
\hline P4 & -19.17 & -24.43 & -26.02 \\
\hline
\end{tabular}

Table-1 shows that, the PSL of Frank is equal to PSL of P2 code and PSL of P3 code is equal to PSL of P4 code. It is also found that Frank code, P2 code have less PSL values when compared with other poly-phase codes. It is known that Frank code, P1 code, P2 codes are applicable only if the code length is a perfect square. Therefore, P3 code and P4 code are used if the length of the code is not a perfect square and this produces relatively lower PSL but higher than Frank code and P2 code.

\section{IV.CONCLUSION}

Since Barker codes are limited in number they are not used in RADAR applications. Poly-phase codes Frank code, P1 code, P2 code, P3 code and P4 code are preferred than bi-phase codes. To avoid masking of targets the value of PSL must be as low as possible. P3 codes and P4 codes are chosen in RADAR applications in multiple target environment.

\section{REFERENCES}

[1] Lewis, B. L., and Kretschmer, F. F., "A New Class of Poly-phase Pulse Compression Codes and Techniques", IEEE Transactions on Aerospace and Electronic Systems, 1981, Vol. AES-17, No. 3, pp. 364-372.

[2] Lewis , B. L., and Kretschmer Jr, F. F., “ Linear Frequency Modulation Derived Poly-phase Pulse Compression Codes", IEEE Transactions on Aerospace and Electronic Systems, 1982, Vol. AES-18, No. 5, pp. 637-641.

[3] Rawat, C. D. and Sarate, A.D., "Effectiveness of Pulse Compression Signal Processing Techniques of RADAR", in Proceedings of International Conference on Advances in Communication and Computing Technologies, 2014, pp. 1-4.

[4] Skolnik M. I., Introduction to RADAR Systems, $3^{\text {rd }}$ Edition, Tata McGraw Hill, 2001.

[5] Richard M. A., Fundamentals of RADAR Signal Processing, Tata McGraw Hill, 2005.

[6] Eustice, D., Baylis, C. and Marks, R. J., "Woodwards Ambiguity Function: from Foundations to Applications", in Proceedings of IEEE Texas Symposium in Wireless and Microwave Circuits and Systems, 2015, pp. 1-17. 